\title{
Efficacité de l'ostéotomie interalvéolaire par piezocision : revue de la littérature
}

\author{
I. Romanet (Aix En Provence), J.H. Catherine (Marseille), P. Laurent (Marseille), R. Lan \\ (Marseille), E. Dubois (Marseille)
}

La durée des traitements orthodontiques est une des plaintes principales des patients en pratique orthodontique, en particulier chez les patients adultes. Le traitement orthodontique chez l'adulte doit s'adapter à des particularités comme l'absence de croissance et l'augmentation des atteintes parodontales. La corticotomie alvéolaire se définit comme une lésion chirurgicale contrôlée de l'os alvéolaire en vue d'induire un remodelage osseux accéléré, responsable localement d'une ostéopénie transitoire facilitatrice du déplacement dentaire. Le recours aux corticotomies alvéolaires permettrait d'accélérer le déplacement dentaire par un facteur 3 ou 4 (2) avec des suites opératoires souvent moins importantes qu'une avulsion de prémolaire, en particulier dans les nouvelles approches mini-invasives. La piezocision permet la correction orthodontique de malocclusions sévères sans présenter les inconvénients des approches chirurgicales extensives et traumatiques des corticotomies alvéolaires classiques. Elle offre un temps chirurgical réduit, des suites post-opératoires minimales, une grande tolérance chez les patients ainsi qu'un parodonte amélioré. (3) Nous présentons une revue de la littérature afin d'évaluer l'efficacité de l'ostéotomie inter alvéolaire par piezocision sur l'accélération ou la facilitation des déplacements dentaires comparée au traitement orthodontique classique. La recherche des données de la littérature a été réalisée sur la base de données pubmed. Les études ont montré que les corticotomies par piezocision favorisent et accélérent les déplacements dentaires avec peu de complications associées. (4) Nous avons retrouvé peu de lésions parodontales et dentaires d'origine iatrogènes. Peu d'études comparatives avec le traitement orthodontique conventionnel ont étés retrouvées mais elles affirment que l'ostéotomie interalvéolaire diminue le temps de traitement orthodontique global de façon significative. Les corticotomies interalvéolaires par piezocision ont un ratio bénéfice risque très favorable et se montre être une solution thérapeutique efficace dans le traitement orthodontique de l'adulte.

1 : Moreau N, Charrier JB. Bone formation and corticotomy induced accelerated bone remodeling: can alveolar corticotomy induce bone formation?. Orthod Fr 2015;86(1):113-120.

2 : Strippoli J., Aknin JJ. Le concept du déplacement dentaire accéléré par corticotomie ou par piézocision, Orthod Fr 2012;83:155回164.

3 : Sebaoun JD, Surmenian J, Dibart S Traitements orthodontique accélérés par piézocision : Une alternative mini-invasive aux corticotomies alvéolaires. Orthod Fr 2011; 82:311-319.

4 : Charrier JB.et al Traitement orthodontique accéléré par corticotomies alvéolaires chez l'adulte. International Orthodontics, 2008;6(4):355-73 